Discussion Paper Series A No.611

\title{
Childcare Availability, Household Structure, and Maternal Employment
}

\author{
Yukiko Asai \\ (Institute of Social Science, University of Tokyo) \\ Ryo Kambayashi \\ (Institute of Economic Research, Hitotsubashi University) \\ Atsuko Tanaka \\ (Department of Economics, University of Calgary) \\ and \\ Shintaro Yamaguchi \\ (Department of Economics, McMaster University)
}

July, 2014

Institute of Economic Research

Hitotsubashi University

Kunitachi, Tokyo, 186-8603 Japan 


\title{
Childcare Availability, Household Structure, and Maternal Employment*
}

\author{
Yukiko Asai $^{\dagger} \quad$ Ryo Kambayashi ${ }^{\ddagger}$ Atsuko Tanaka ${ }^{\S}$ Shintaro Yamaguchi ${ }^{\circledR}$ \\ July 4th, 2014
}

\section{Preliminary Draft}

\begin{abstract}
We estimate the causal effects of childcare availability on the maternal employment rate using prefecture panel data constructed from the Japanese quinquennial census 1990-2010. We depart from previous contributions by controlling for prefecture fixed effects, without which the estimates can be severely biased upward. We find that the treatment effect is heterogeneous: the employment rate of mothers in nuclear households increases with childcare availability, while that of mothers in three-generation households does not. We apply our estimates to decomposition of the growth of the maternal employment rate from 1990 to 2010. The decomposition indicates that the increase in childcare availability raised the maternal employment rate by about two percentage points. We also find that dissolution of three-generation household lowered the maternal employment rate. This negative effect is more strongly pronounced in small prefectures where the household structure changed dramatically.
\end{abstract}

Keywords: childcare; female labor supply; maternal employment; nuclear family; three-generation family

JEL Codes: J13, J21, J22

${ }^{*}$ The authors thank the financial support from Joint Usage and Research Center, Institute of Economic Research, Hitotsubashi University. Tanaka acknowledges the use of the data from Japanese Panel Survey of Consumers conducted by the Institute for Research on Household Economics.

${ }^{\dagger}$ Institute of Social Science, University of Tokyo

Institute of Economic Research, Hitotsubashi University

$\S$ Department of Economics, University of Calgary

${ }^{\mathbb{I}}$ Corresponding author. Department of Economics, McMaster University, 1280 Main St. W., Hamilton, ON. Canada L8S 4M4. Email: yamtaro@mcmaster.ca. 


\section{Introduction}

Maternal employment hinges on quality, affordability, and availability of childcare. In countries where childcare market is regulated and heavily subsidized, high quality and affordable childcare service is provided, but the supply often falls short of the demand. Because supporting working mothers is expected to raise the low fertility rate, the Japanese government has implemented a series of pro-working-mother policies including an expansion of capacity of accredited childcare centers since early 1990s. In this paper, we estimate the causal effects of childcare availability on the maternal employment, using the prefecture panel data from the Japanese quinquennial Census 1990-2010.

In North America, the childcare service is provided by the private sector and the focus of the research has been the elasticity of maternal labor supply with respect to the childcare price ${ }^{1}$ In other parts of the world, childcare is regulated and heavily subsidized, and hence, availability is more of an issue. ${ }^{2}$ Circumstances for childcare services in those countries are more comparable to Japan. Most previous studies from Japan on childcare availability and a female labor market outcome rely on cross-sectional variation between prefectures. ${ }^{3}$. While the reported strong correlation is suggestive, it may not necessarily imply the causal effects of childcare availability on the female labor market outcome. If unobserved characteristics of prefectures are positively correlated with childcare availability and maternal employment, the estimates for the causal effects are biased upward.

We depart from the previous contributions from Japan by controlling for the prefecture fixed effects using the prefecture panel data. Using the ratio of childcare capacity to population of children under 6 as a proxy for childcare availability, we estimate the causal effects of childcare availability on the maternal employment rate by the first-difference regression and OLS with prefecture and year fixed effects. The main finding is that the effect of childcare availability on maternal employment is heterogeneous across household types. Specifically, mothers in nuclear households increase their labor supply with childcare availability, while mothers in three-generation households do not, because informal childcare from grand parents is available for three-generation households. Our main result is robust to inclusion of a prefecture-specific trend in the maternal employment rate and choice of econometric models.

We address a potential simultaneity bias due to inter-prefecture migration for childcare, using data from Japanese Panel Survey of Consumers (JPSC). We take a sample of mothers who have

\footnotetext{
${ }^{1}$ See Blau and Robins (1988), Connelly (1992), Michalopoulos, Robins, and Garfinkel (1992), and Kimmel (1998), for example.

${ }^{2}$ See Berlinski and Galiani (2007), Lefebvre and Merrigan (2008), and Brilli, Del Boca, and Pronzato (2013), for example.

${ }^{3}$ See Shigeno and Ookusa (1999), Higuchi, Matsuura, and Sato (2007), Unayama (2011), and Abe (2013), for example.
} 
children under 6 and find that at most $1 \%$ of them moved from other prefecture for better childcare availability at the most recent move. Given that inter-prefecture migration for childcare is rare, we do not concern about the simultaneity bias.

We apply our estimates to decomposition of the maternal employment growth from 1990 to 2010. We analyze large and small prefectures separately. The former consists of the eight most densely populated prefectures including Tokyo, and the latter is the rest of Japan. We find that the growth of childcare availability raised the maternal employment rate up to about two percentage points in both prefecture groups, but childcare availability improved for very different reasons. Note that childcare availability improves either by an increase of capacity or by a decrease of child population. In the large prefectures, the increase of childcare capacity accounts for more than a half of the growth of childcare availability. On the other hand, in the small prefectures, the growth of childcare availability is mostly explained by the decreasing child population.

The share of nuclear households increased in both prefecture groups, which lowered the maternal employment rate, because informal childcare by grand parents is not available for nuclear households. This negative effect is particularly strong and lowered the maternal employment rate by up to four percentage points in the small prefectures, because three-generation households dissolved more rapidly.

This paper contributes to the literature on childcare and labor supply of mothers. Earlier studies on the U.S. including Blau and Robins (1988), Connelly (1992), Michalopoulos, Robins, and Garfinkel (1992), and Kimmel (1998) focus on the elasticity of maternal labor supply with respect to the price of childcare services. These papers construct the childcare price from observed expenditures either at the household level or at the region level. This approach may suffer a bias because the constructed childcare price variable is likely to be endogenous. Gelbach (2002) avoids this problem and estimates the causal effect by taking schooling for young children as a large implicit subsidy for childcare.

In Europe and other countries where childcare service is regulated and heavily subsidized, studies focus on the effect of a large subsidy or availability of subsidized public childcare. The approach taken by these papers is comparable to that by Gelbach (2002). Berlinski and Galiani (2007) estimate the effect of expansion of preschools on maternal labor supply in Argentina. Lefebvre and Merrigan (2008) find a positive maternal employment effect of Quebec's five-dollars-a-day childcare policy. Brilli, Del Boca, and Pronzato (2013) find a positive effect of childcare availability and mother's work in Italy. Similar to these countries, childcare in Japan is regulated and heavily subsidized. Our paper is close to these non-U.S. studies, not only because childcare is heavily subsidized in Japan, but also because our identification of the causal effect relies on variations in the growth of childcare availability across prefectures.

Most previous studies on Japan use cross-section data. Examples include, but not limited to, 
Shigeno and Ookusa (1999), Higuchi, Matsuura, and Sato (2007), Unayama (2011), and Abe (2013). These papers present a positive correlation between childcare availability and female employment, but this correlation may not be interpreted as the causal effect of childcare, because unobserved prefecture characteristics may cause an endogeneity bias. Indeed, we find that the estimated coefficient for childcare availability is upward-biased by $50 \%$ when the prefecture fixed effects are omitted.

Recently, Lee and Lee (2014) have taken a very different approach to identification of the causal relationship. Using aggregate data at the country level on childcare availability, female labor force participation rate, and fertility rate from 1971 to 2009, they try to establish Granger-causality using a time-series econometrics technique. Our identification strategy is very different from Lee and Lee (2014) in that we use variations in the growth of childcare availability across prefectures. Moreover, Lee and Lee (2014) do not include the household structure or the nuclear household share in their VAR model. Our analysis shows that the household structure strongly affects the maternal employment rate and has changed over time significantly. Omitting this variable in the VAR model might affect their estimation results.

The rest of the paper is structured as follows. In Section 2, we describe the institutional background and data. In Section 3, we establish stylized facts on childcare availability and maternal employment. Section 4 lays out the econometric model and shows our estimation results. We also discuss robustness of the result there. In Section 5, we discuss potential sources of a bias for our results. In Section 6, we apply our estimates to study the roles of childcare availability and the household structure on the employment growth of mothers. We conclude in Section 7.

\section{Background and Data}

\subsection{Childcare Policies in Japan}

Childcare service is strictly regulated in Japan. For a childcare center to be accredited and subsidized by the national and municipality governments, it must satisfy the criteria about capacity, area and teacher per pupil, etc. Because accredited childcare centers are subsidized, the average monthly fee for a child is low at 25,556 JPY (about 250 USD) ${ }^{4}$ although different fees are charged depending on regions and income of the household. Non-accredited childcare centers satisfy lower quality standards than those for accredited childcare centers. While some municipality governments subsidize non-accredited centers, the national government does not subsidize them, which makes their fee higher than that for accredited childcare centers. Their average monthly fee for a child age 0 is 46,330 JPY (about 460 USD) and that for child age 5 is 34,161 JPY (about 340

\footnotetext{
${ }^{4}$ See Table 7 on page 8 of Ministry of Health and Wealth (2009)
} 
USD) ${ }^{5}$

According to Comprehensive Survey of Living Conditions 2010, ${ }^{6} 92 \%$ of childcare centers are accredited and the remaining $8 \%$ are non-accredited. Because non-accredited childcare centers are not subsidized and at lower quality, they are uncommon except for the large prefectures such as Tokyo and Kanagawa where the supply of accredited childcare is exceeded by the demand. For example, the share of non-accredited childcare centers is $10 \%$ in Tokyo and 16\% in Kanagawa, while it is nearly zero in small prefectures including Gunma, Toyama, Fukui, Yamanashi, Nagano, Wakayama, and Tottori.

Insufficient supply of accredited childcare has been considered an obstacle for mothers' market work and a cause of the low fertility rate in Japan. The Japanese government implemented a series of policies for raising the fertility rate since the mid 90's. The Angel Plan (1994-1998) and the New Angel Plan (1999-2003) are agreements between ministers and intended to support working mothers and include expansion of childcare capacity, extending childcare service hours, opening childcare facilities on weekends and holidays, and subsidies to promote parental leave take-up and shorter working hours. However, these plans do not have legally binding power, and consequently, they failed to increase the capacity of accredited childcare. Indeed, it decreased from 1990 to 2000 by $10 \%$ even though childcare capacity per child population slightly increased due to the low fertility rate. The Japanese government enacted Basic Act for Measures to Cope with Society with Declining Birthrate in 2003, which is legally binding. Since then, the capacity of accredited childcare significantly and steadily increased: childcare capacity increased by $12.2 \%$ from 2000 to 2010.

\subsection{Measuring Childcare Availability}

Our key explanatory variable is an index of childcare availability at the prefecture level, which is defined as the ratio of the capacity of accredited childcare to the population of children under 6. As we explained above, the share of accredited childcare centers is $92 \%$ in 2010 , and hence, the proposed index roughly measures overall availability of center-based childcare. In this paper, childcare capacity and enrollment statistics cover accredited childcare centers only, unless otherwise noted. We draw childcare capacity data from annual Report on Social Welfare Administration and Services ${ }^{7}$ and child population data from the Japanese quinquennial census 1990-2010. Some of previous studies measure enrollment rate (the proportion of those enrolled in childcare among children under 6), but it may be correlated not only with supply of childcare, but also with demand

\footnotetext{
${ }^{5}$ See Table 12 on page 14 of Ministry of Health and Wealth (2009)

${ }^{6}$ See Table 18 in Volume 3.

${ }^{7}$ Childcare capacity data are also available from Survey on Social Welfare, but data are not comparable before and after 2007.
} 
for childcare. This issue questions interpretation of the index as an availability measure. Our measure of childcare availability is based on capacity, which is a supply side factor, and hence, we safely interpret our index as a measure of childcare availability.

Alternative measure of childcare availability is the number (or proportion) of children on the waiting list for accredited childcare, but there are at least two reasons why we do not use it. First, in 2003, the government changed the method to count the number of waitlisted children, which makes the statistics before and after 2003 not comparable. Second, whether being waitlisted or not is endogenous. If parents expect a very low chance of getting accepted by an accredited childcare center, they are unlikely to submit an application to avoid a time cost of application. If this is the case, the number of waitlisted children also picks up demand side factors. Indeed, it is reported that city of Yokohama experienced a significant rise in the number of applications immediately after the year when Yokohama declared that no children in Yokohama were waitlisted. This anecdote suggests a large potential demand greater than the number of waitlisted children.

Yet another possible measure of childcare availability is proposed by Unayama (2011) who studies the relationship between job continuity at marriage and childcare availability. His index is the ratio of childcare capacity to the number of women age 20-39. His rationale is that the number of women age 20-39 measures the potential number of children. Our index based on the actual number of children directly measures the current availability, which we think is more relevant for decisions by mothers who currently have children under 6 .

\subsection{Household and Prefecture Characteristics}

We draw our data on household and prefecture characteristics from the Japanese quinquennial census from 1990 to $2010 .^{8}$ Our analysis focuses on households with two parents and children under age 6 . These households are categorized into three groups by the census. The first household type is a nuclear household in which only two parents and children reside. The second household type is a three-generation household that consists of two parents, children, and grand parents. The third household type is other household that consists of two parents, children, and other adult such as relatives. This last type is only about $0.02 \%$ of all two-parent household with children under 6 .

In the census, employment is identified by whether an individual did a paid work or not from September 24th to 30th of the year. If an individual did not work but was on leave, she/he is considered employed by the census. Employment status of head (which is a man by definition) and wife of the household is reported. We also calculate the average ages of the head and wife, although we cannot observe the joint distribution of their employment status and age at the prefecture level.

As a measure of local economic condition, we construct a local unemployment rate for indi-

\footnotetext{
${ }^{8}$ All of our data from the census 1990-2010 are publicly available and downloadable from www.e-stat.go.jp.
} 
viduals age 15 and over at the prefecture level.

\subsection{Summary Statistics}

Table 1 reports mean of each variables by year and household type using the number of households as weight. Our childcare availability index, which is defined by the ratio of capacity to population of children under 6, steadily increases over time from 0.248 in 1990 to 0.338 in 2010 for the pooled sample that include all types of household. The average of the capacity-population ratio for nuclear households is lower than that for three-generation households, which reflects the fact that the share of nuclear households is higher in the metropolitan area where childcare service is less available.

Table 1: Characteristics of Prefecture and Household with Children Under 6

\begin{tabular}{lrrrrr}
\hline \hline & 1990 & 1995 & 2000 & 2005 & 2010 \\
\hline All HH & & & & & \\
Cap./U6-Pop. & 0.248 & 0.265 & 0.269 & 0.302 & 0.338 \\
\% Employed & 0.341 & 0.329 & 0.334 & 0.372 & 0.412 \\
Age & 31.580 & 31.757 & 32.142 & 32.883 & 33.720 \\
Age of Husband & 34.454 & 34.517 & 34.564 & 34.953 & 35.632 \\
\% Husband Empl. & 0.988 & 0.985 & 0.976 & 0.965 & 0.941 \\
Unemp. Rate & 0.030 & 0.043 & 0.048 & 0.060 & 0.064 \\
\% Nuclear HH & 0.712 & 0.752 & 0.804 & 0.837 & 0.865 \\
\hline Nuclear HH & & & & & \\
Cap./U6-Pop. & 0.241 & 0.258 & 0.263 & 0.296 & 0.332 \\
\% Employed & 0.274 & 0.280 & 0.300 & 0.347 & 0.396 \\
Age & 31.560 & 31.632 & 32.049 & 32.886 & 33.763 \\
Age of Husband & 34.373 & 34.313 & 34.382 & 34.879 & 35.614 \\
\% Husband Empl. & 0.988 & 0.984 & 0.976 & 0.965 & 0.941 \\
Unemp. Rate & 0.031 & 0.044 & 0.048 & 0.060 & 0.064 \\
\hline 3-Generation HH & & & & & \\
Cap./U6-Pop. & 0.266 & 0.285 & 0.293 & 0.331 & 0.376 \\
\% Employed & 0.511 & 0.478 & 0.476 & 0.499 & 0.517 \\
Age & 31.628 & 32.147 & 32.537 & 32.884 & 33.458 \\
Age of Husband & 34.652 & 35.146 & 35.327 & 35.346 & 35.750 \\
\% Husband Empl. & 0.990 & 0.986 & 0.975 & 0.968 & 0.945 \\
Unemp. Rate & 0.028 & 0.039 & 0.044 & 0.057 & 0.064 \\
\hline & & & & & \\
\hline
\end{tabular}

Source: Census 1990-2010.

Note: Unit of observation is prefecture. The number of households is used as weight.

The employment rate of mothers with children under 6 rose from $34.1 \%$ in 1990 to $41.2 \%$ 
in 2010. This increase is largely driven by the growing employment rate of mothers in nuclear households: it increased from $27.4 \%$ in 1990 to $39.6 \%$ in 2010. Although the employment rate of mothers in three-generation households is as high as $51.1 \%$ in 1990 , it decreased to $47.8 \%$ in 1995 and gradually increased to $51.7 \%$ in 2010 . Essentially the maternal employment rate for three-generation households grew very little from 1990 to 2010.

The employment rate of the husbands decreased from $98.8 \%$ in 1990 to $94.1 \%$ in 2010 . In contrast to women, we find little difference in male employment rate across household type.

The average age of mothers increased from 31.580 in 1990 to 33.720 in 2010. That of husband also increased from 34.454 in 1990 to 35.632 in 2010. The rise in parents' age reflects the fact that women delayed childbirth in the past few decades. We find little difference in parents' age across household type.

The average local unemployment rate rose from $3 \%$ in 1990 to $6.4 \%$ in 2010. Despite that nuclear and three-generation households tend to reside in different regions, we find little difference in local unemployment rates that nuclear and three-generation households face.

\section{Stylized Facts}

In this section we show sets of scatter plots of the maternal employment rate and our measure of childcare availability. The objective of the scatter plots is to clarify what variation of the data enables us to identify the causal effect of childcare availability on maternal employment. They also help us examine if the regression results are not driven by outliers or by data from specific years. Our informal analysis using the scatter plots are followed by more rigorous regression analysis in the next section.

Using Japanese data, several papers including Shigeno and Ookusa (1999), Higuchi, Matsuura, and Sato (2007), Unayama (2011), and Abe (2013) find a positive correlation of childcare availability and women's work. The correlation of the two variables may be driven by unobserved prefecture characteristics, and hence, it may not be interpreted as a causal effect of childcare availability on the female labor market outcome. To address this issue, we plot changes in childcare availability and changes in maternal employment over a five-year period. By looking at the changes, instead of the levels, we can remove unobserved prefecture characteristics that are constant over the five-year period. The prefecture fixed effects may include preference for maternal work, industry structure, commuting time, etc.

Figure 1 shows scatter plots of 5-year changes in the capacity-population ratio and the maternal employment rate for nuclear households that consist of parents and children only. The positive correlation is clearly seen in all periods and the correlation is statistically significant. The scatter plots also show that the correlation is not driven by outliers and that there is significant variation in 
changes in the capacity-population ratio across prefectures. Figure 1 provides prima facie evidence for the effect of childcare on maternal employment for nuclear households.

Figure 2 shows scatter plots for three-generation households that consist of grand parents, parents, and children. ${ }^{9}$ For three-generation households, no clear relationship is seen. Indeed, the correlation for 1995-2000 is significantly negative, while that for 2005-2010 is significantly positive.

The heterogeneous responses to childcare availability presented in this subsection are intuitive. For nuclear households, maternal employment hinges on whether childcare service is available or not. In contrast, for three-generation households, availability of childcare is irrelevant for maternal employment, because they rely on informal childcare by grandparents. It is true that many previous papers (e.g. Sasaki (2002)) point out that mothers in three-generation households are more likely to participate in the labor market than those in nuclear households, but the effect of childcare availability is usually assumed homogeneous. Allowing for heterogeneous treatment effects between nuclear and three-generation households is important for prediction, because the share of nuclear households varies across years and prefectures significantly.

\footnotetext{
${ }^{9}$ Other individuals such as relatives may or may not live together.
} 

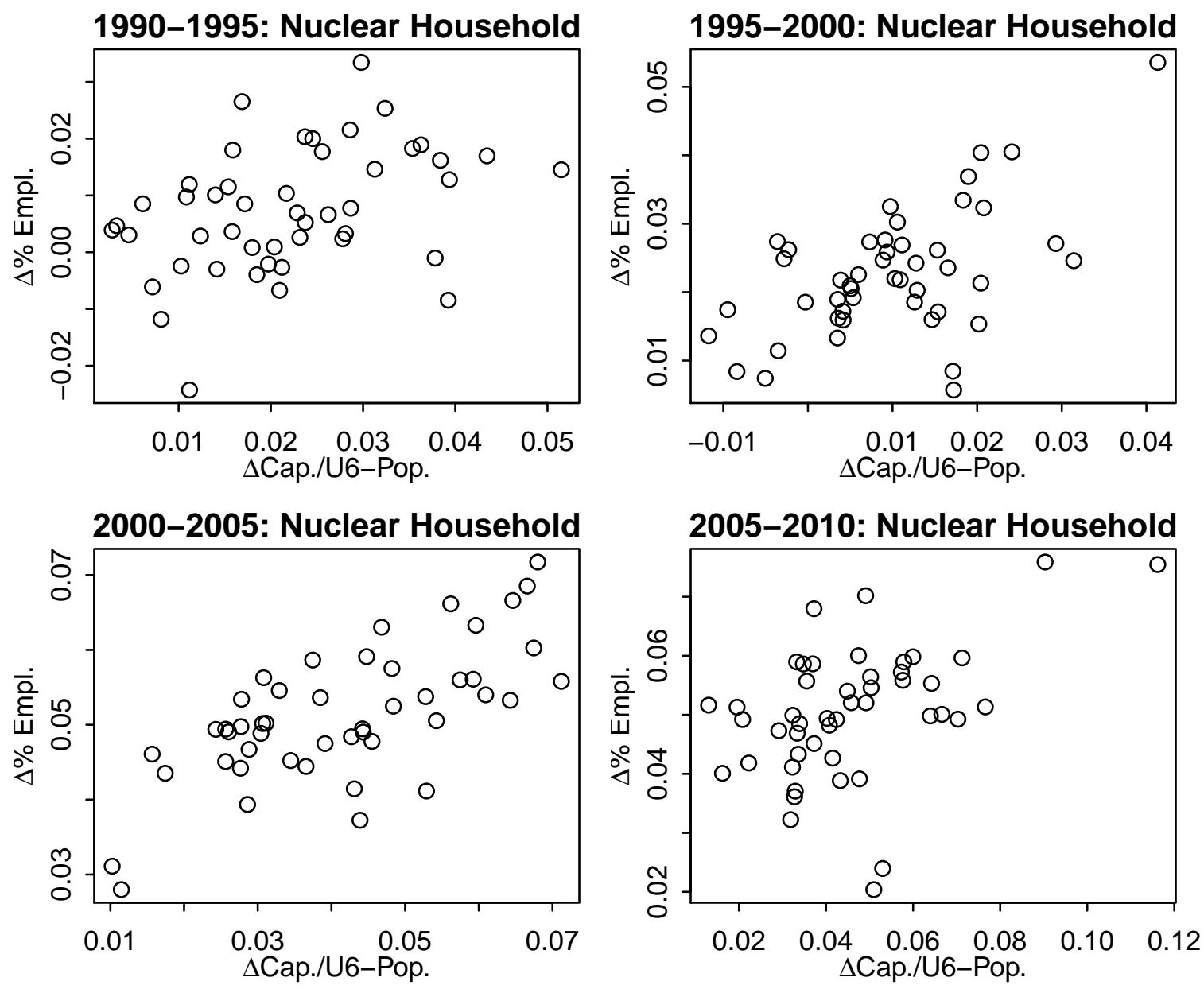

Source: Census 1990-2005

Note: Unit of observation is prefecture. The vertical axis shows 5-year changes in the employment rate of mothers in nuclear households. The horizontal axis shows 5-year changes in the capacitypopulation ratio.

Figure 1: Changes in Childcare Availability and Maternal Employment Rate (Nuclear Household) 

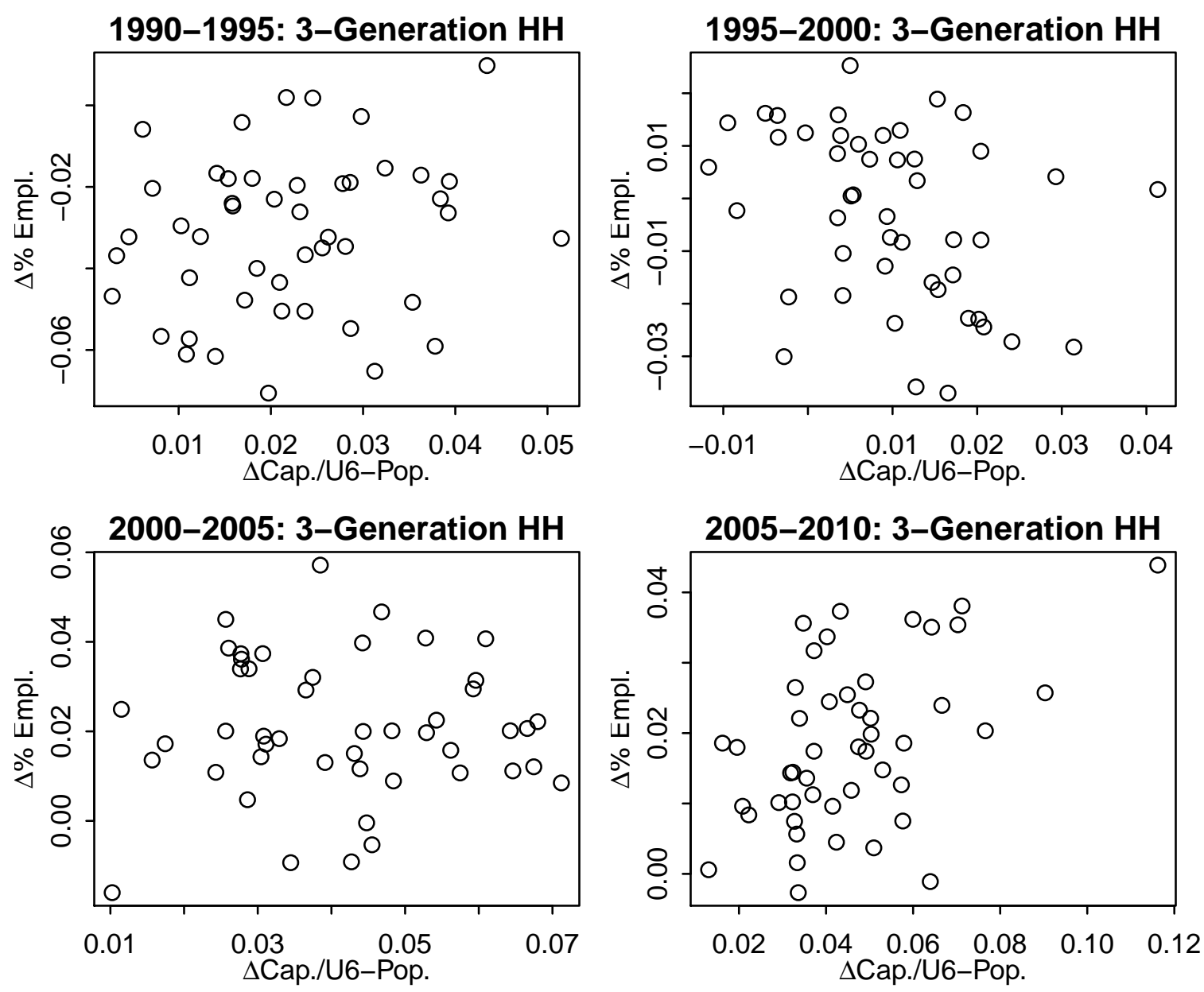

Source: Census 1990-2005

Note: Unit of observation is prefecture. The vertical axis shows 5-year changes in the employment rate of mothers in three-generation households. The horizontal axis shows 5-year changes in the capacity-population ratio.

Figure 2: Changes in Childcare Availability and Maternal Employment Rate (Three-Generation Household)

\section{Effects of Childcare Availability on Maternal Employment}

\subsection{Econometric Model}

We now turn to further analysis of the causal effects of childcare availability using regression models. Consider the following econometric model

$$
Y_{i t}=\beta_{0}+\beta_{1} C C A_{i t}+\beta_{2} X_{i t}+\theta_{i}+\xi_{t}+\varepsilon_{i t},
$$


where $Y_{i t}$ is a measure of outcome such as the maternal employment rate in prefecture $i$ in year $t, C C A_{i t}$ is our measure of childcare availability given by the ratio of capacity to child population, $X_{i t}$ is a vector of observed characteristics of household and prefecture, $\theta_{i}$ is prefecture fixed effects, $\xi_{t}$ is year fixed effects, and $\varepsilon_{i t}$ is an error term that is uncorrelated with other variables. The prefecture fixed effects capture unobserved household and prefecture characteristics including preference for maternal employment. The year fixed effects capture factors that affect maternal employment equally across prefecture. For example, the effects of changes in parental leave and other legislation ${ }^{10}$ are included in the year fixed effects. The year fixed effects also account for the effects of a nationwide secular rise in female wages. We estimate the coefficient for childcare availability $\beta_{1}$ by the first difference estimator and OLS with prefecture and year fixed effects. In all regression models, we use the number of households as weights.

\subsection{Regression Results}

We examine if childcare availability increases maternal employment based on regression models. Our dependent variable is 5-year differences in the maternal employment rate. The main explanatory variable is the ratio of capacity to child population as a proxy for childcare availability. We pool observations across years, but no specific period drives the results, as evidenced by the scatter plots in Figures 1-2.

Table 2 reports the results for the first-difference estimator. The population for Models 1 through 4 is mothers of children under 6 in nuclear households. When the 5-year changes are stacked and regressed without an additional control, the coefficient is 0.775 (see Model 1). Once year dummies are included as a control, the coefficient drops to 0.352 (see Model 2), which suggests that both maternal employment and childcare availability increase over time. As additional control variables, we include in the regression the average age of mothers, that of their husbands, and the local unemployment rate at the prefecture level. Adding these controls modestly lowers the coefficient of interest to 0.316 (see Model 3).

To assess the extent of the endogeneity bias due to the prefecture fixed effects, we estimate Equation (1) by OLS with year and prefecture fixed effects. Table 3 reports the OLS estimation results. Model 1 includes no covariates other than the capacity-population ratio, and the estimated coefficient is 0.668 . It decreases to 0.572 in Model 2 in which only year dummies are added. Model 3 adds prefecture dummies to Model 2: the coefficient drops from 0.572 to 0.391 , which implies that the coefficient is upward-biased by $46 \%$ if prefecture fixed effects are omitted. This result warns us that omitting prefecture fixed effects can bias the estimates for the effects of childcare availability significantly.

\footnotetext{
${ }^{10}$ See Asai (2012) and Yamaguchi (2014) for the employment effects of parental leave legislation.
} 
We also examine the employment effects of childcare availability for mothers in three-generation households. As already suggested by the scatter plot in Figure 2, the employment of mothers in three-generation households does not respond to childcare availability (see Model 5 in Table 2), because informal childcare from grand parents may be available. Evidence from Comprehensive Survey of Living Conditions 2010 indicates that the share of children who are looked after by grand parents ${ }^{11}$ tends to be higher in prefectures where the share of three-generation households is high. The correlation coefficient for the two is as high as 0.80 , which suggests that children in three-generation households are likely to be looked after by their grand parents and the mothers employment does not hinge on availability of childcare.

In Models 6-8 in Table 2, we study employment of all mothers who have children under 6 regardless of household types. When the change in the nuclear household share is omitted, the coefficient for the capacity-population ratio is essentially zero (see Model 6). When it is included, the coefficient is positive and significant (see Model 7). This coefficient is average over different household types, but the results for Models 3 and 5 indicate that the effects are very different between nuclear and three-generation households. To account for the heterogeneity, we include an interaction term of the capacity-population ratio and the nuclear household share at the beginning of the five-year period. The coefficient for the interaction term is positive and significant as expected (see Model 8).

We close this subsection by summarizing our main findings. First, the employment rate of mothers in nuclear households increases with childcare availability. Second, the employment rate of mothers in three-generation households do not respond to childcare availability. Third, omitting prefecture fixed effects severely biases the estimated causal effects upward.

\subsubsection{Robustness}

Our estimates might potentially be biased because we have a small number of control variables and omitted variables might generate a prefecture-specific trend in the maternal employment rate. We address this concern by allowing for prefecture fixed effects for 5-year changes in the maternal employment rate. The result for Model 4 in Table 2 indicates that allowing for a prefecture-specific trend does not change the coefficient for the childcare availability measure from our baseline specification of Model 3.

Another concern is misspecification. Equation (1) can be estimated by OLS with year and prefecture fixed effects. The first-difference estimator and OLS with the fixed effects are based on different assumptions, and hence, we should examine the consequences of different statistical assumptions. Table 3 shows the results from OLS with the fixed effects. Model 4 in Table 3 and

\footnotetext{
${ }^{11}$ See Table 18 in Volume 3.
} 


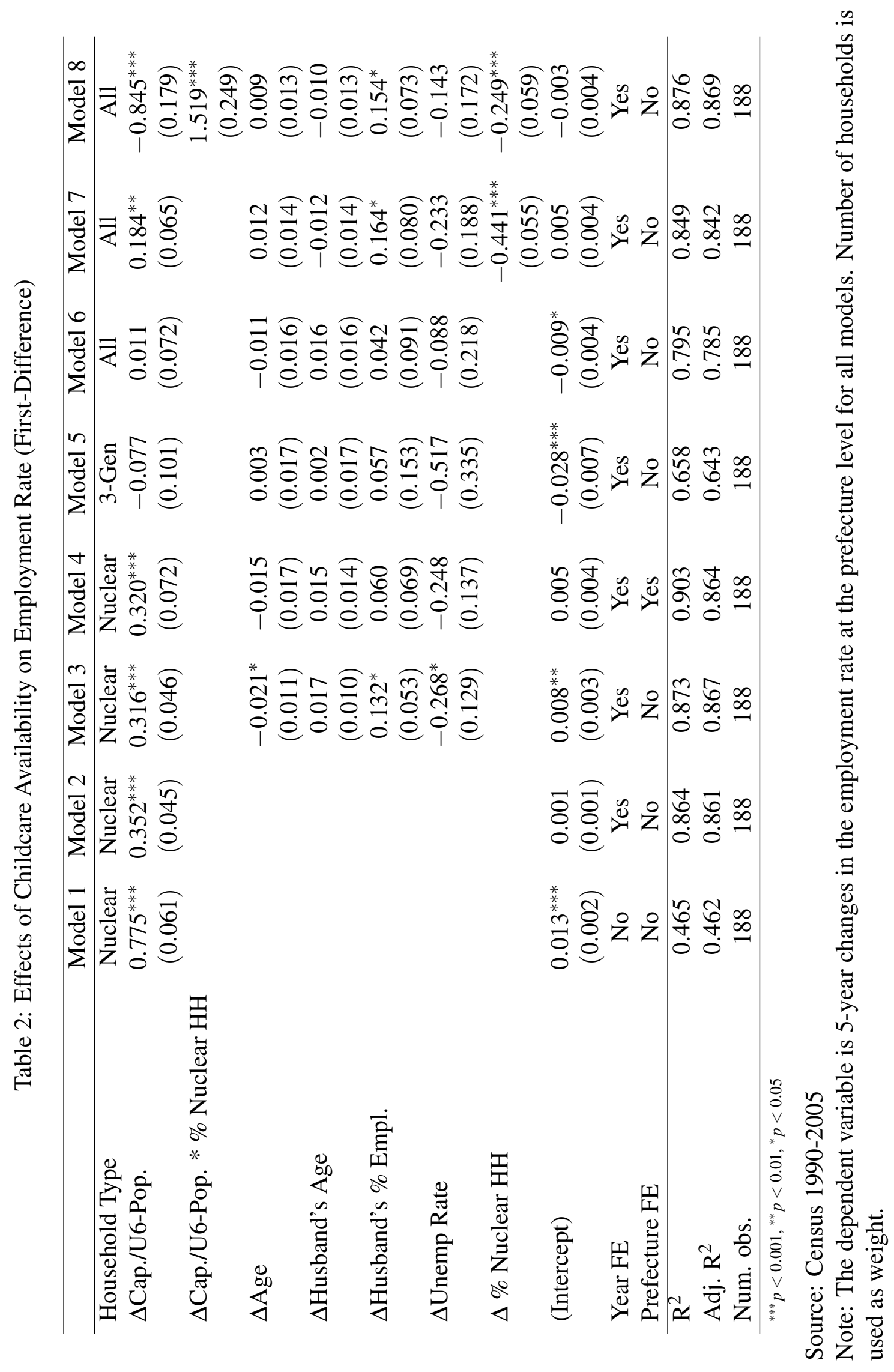




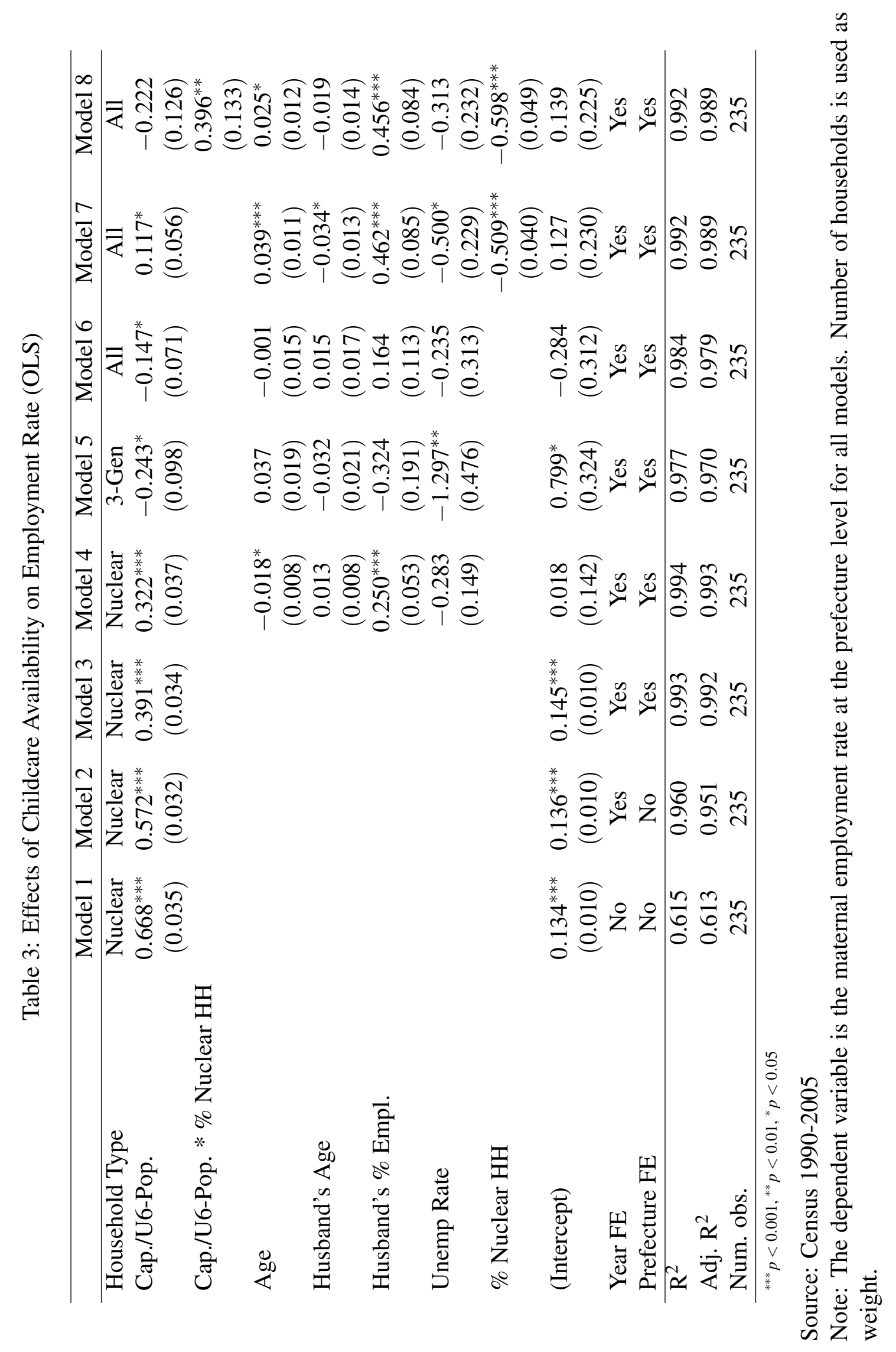


Model 3 in Table 2 are comparable, and their coefficients for the capacity-population ratio are remarkably similar ( 0.322 for OLS with the fixed effects and 0.316 for the first-difference model).

An interesting difference between OLS with the fixed effects and the first-difference regression is found for mothers in three-generation households. The result for OLS with the fixed effects implies that the maternal employment rate for three-generation households decreases with childcare availability (see Model 5 in Table 3). Although this is not robust as not seen in the first-difference model (see Model 5 in Table 2), it might suggest that more labor supply of mothers in nuclear households crowded out mothers in three-generation households.

We show that our result for nuclear households is robust to inclusion of a prefecture-specific trend and choice of regression models. The result for three-generation households is less robust, but the bottom line is that mothers in three-generation households do not increase their labor supply in response to childcare availability.

\subsubsection{Childcare Enrollment}

Our hypothesis is that insufficient supply of childcare service prevents Japanese mothers from participating in the labor force. If this is true, we should observe not only more working mothers, but also more children enrolled in accredited childcare facilities, when childcare becomes more available. If we do not observe more enrollment, the increase in maternal employment is unlikely to be caused by more childcare availability despite the positive result above. We examine if childcare enrollment, as an intermediate outcome for maternal employment, responds to childcare availability as we expect.

We regress 5-year changes in the childcare enrollment rate on 5-year changes in the capacitypopulation ratio and other control variables. The enrollment rate is defined as the ratio of the number of children enrolled in accredited childcare centers to the population of children under 6 in a given prefecture. We do not observe the household characteristics of these children.

Table 4 reports the regression results. Without a control, the coefficient for the capacity rate is 0.878 (see Model 1), implying that one newly available spot is filled by 0.878 children in a fiveyear period on average. Although the estimate is less than one, it is not significantly different from one. The coefficient rises to 1.202 if year fixed effects are controlled (see Model 2). Including changes in ages of mother and father, the nuclear household share, and the local unemployment rate does not have any additional effect on the coefficient (see Model 3).

We also allow for different trends in the childcare enrollment rate across prefectures, in order to ensure that the change in the enrollment rate is not entirely driven by a prefecture-specific trend. Once prefecture fixed effects are included, the coefficient changes to 0.989 (see Model 4). Interestingly, the coefficient for the change in the nuclear household share is also positive and significant. This is intuitive, because informal childcare from grand parents is unlikely to 
be available for nuclear households. The estimated coefficient for the change in the capacitypopulation ratio is not significantly different from one, implying that one additional spot leads to one more child enrolled in childcare facilities. This result is consistent with the fact that the supply of childcare service falls short of the demand in Japan.

Table 4: Effects of Childcare Availability on Childcare Enrollment Rate (First-Difference)

\begin{tabular}{lcccc}
\hline & Model 1 & Model 2 & Model 3 & Model 4 \\
\hline$\Delta$ Cap./U6-Pop. & $0.878^{* * *}$ & $1.202^{* * *}$ & $1.166^{* * *}$ & $0.989^{* * *}$ \\
& $(0.086)$ & $(0.074)$ & $(0.072)$ & $(0.097)$ \\
$\Delta$ Mother's Age & & & -0.007 & 0.023 \\
& & & $(0.016)$ & $(0.022)$ \\
$\Delta$ Father's Age & & & -0.027 & $-0.049^{*}$ \\
& & & $(0.016)$ & $(0.019)$ \\
$\Delta \%$ Nuclear HH & & & -0.013 & $0.405^{* *}$ \\
& & & $(0.061)$ & $(0.142)$ \\
$\Delta$ Unemp Rate & & & 0.309 & 0.361 \\
& & & $(0.210)$ & $(0.212)$ \\
(Intercept) & $0.024^{* * *}$ & -0.004 & -0.003 & $-0.028^{* *}$ \\
& $(0.003)$ & $(0.002)$ & $(0.004)$ & $(0.009)$ \\
Year FE & No & Yes & Yes & Yes \\
Prefecture FE & No & No & No & Yes \\
\hline $\mathrm{R}^{2}$ & 0.362 & 0.773 & 0.823 & 0.884 \\
Adj. R & 0.358 & 0.768 & 0.815 & 0.836 \\
Num. obs. & 188 & 188 & 188 & 188 \\
\hline${ }^{* * *} p<0.001,{ }^{* *} p<0.01,{ }^{*} p<0.05$ & & &
\end{tabular}

Source: Census 1990-2005

Note: The dependent variable is 5-year changes in the childcare enrollment rate at the prefecture level for all models. Average ages of mother and father are for all two-parents household with children under age 6 including nuclear, three-generation, and other households. Local unemployment rate is for all individuals age 15 and over in the prefecture. Population of children under 6 is used as weight.

\section{Potential Biases}

\subsection{Inter-Prefecture Migration}

Although the first-difference regression and OLS with the fixed effects remove prefecture fixed effects, there might be a simultaneity bias. Namely, there may be a correlation between childcare availability and mothers' unobserved willingness to work even after time-invariant household and prefecture characteristics are controlled for. The most plausible source of the simultaneity bias 
is existence of households moving to a prefecture in which childcare service is more available. Popular narrative says that obtaining a spot in an accredited childcare center is extremely hard in Tokyo and that some people even move to other municipality for childcare. For example, in the article from New York Times, Tabuchi (2013) reports, "Some families are so anxious to get into public day care that they upend their lives, moving to districts known to have the shortest waiting lists." However, very little statistical evidence on this issue has been reported.

We provide evidence that households do not move for childcare, using information on moving from JPSC. JPSC has started in 1993 with 1,500 women age 24-34, and added a new cohort of 500 women age 24-27 in 1997. JPSC asks these two cohorts about their last five moves, but we focus on the most recent move to avoid a potential recall bias. We analyze the 1993 and 1997 cohorts separately, because JPSC asks slightly different questions. Note that only inter-prefecture moves matter to our analysis, because all of our variables are at the prefecture level.

We first examine a sample of 721 women from the 1993 cohort who have children under 6 at the time of the survey in October 1993. Out of 721, 118 women moved from other prefecture than the current one at the most recent move. Out of 118 , only 15 women answered that they knew or researched about childcare availability at the current location before moving in. Note that knowing about childcare availability does not necessarily mean that they move for childcare availability. The survey further asks the respondents to choose the closest one that describes the reason of the move from twelve alternatives. Although "child rearing and education" is not in the 1993 survey, we can still exclude answers that are clearly unrelated to child rearing and education. After we exclude 6 individuals who moved for attending school or husband's job transfer, we have 9 individuals who may have moved for childcare availability. This implies that at most only $1 \%$ (9 out of 721) of mothers moved for more childcare availability.

We also examine a slightly different subpopulation from the 1993 cohort. Specifically, we take a sample of 293 women who had children under 6 at the time of the most recent move (instead of at the time of the survey). Note that this is a sample of movers. Examining this subpopulation sheds light on the reasons of move for households with young children. Out of 293, 49 women (17\% of movers) moved from other prefectures. Out of the 49 inter-prefecture movers, only 11 women ( $22 \%$ of inter-prefecture movers) knew or researched about childcare availability before their move. After we exclude 4 individuals who moved for husband's job transfer, we are left with 7 individuals. This implies that at most only $2 \%$ of movers might have moved for childcare availability.

We turn to the analysis of the 1997 cohort. JPSC asks this cohort if they moved for child rearing or education. There are 133 women who have children under 6 in the 1997 cohort. Out of 133, only 6 women answered that they moved for child rearing or education. We also examine a mover sample from the 1997 cohort. Our sample of movers include 63 women who had children under 6 
at the time of the most recent mover (instead at the time of the survey). Out of 63 , only 6 (9\% of movers) answered that she moved for child rearing and/or child education. ${ }^{12}$

Our analysis of two cohorts in the JPSC suggests that inter-prefecture migration for childcare is quite uncommon, despite that media often reports interviews of parents who are desperate for the accredited childcare service. This might look somewhat surprising for some readers, but we consider this is reasonable given that the cost of an inter-prefecture move can be high. The cost of inter-prefecture moving includes not only moving expenses but also the loss of social networks, familiarity with the local public services, proximity to the current workplace, etc. Perhaps, moving for childcare is beneficial for highly skilled women despite the significant cost, but only $21 \%$ of married women age 30-34 have bachelor's degree or higher, according to the Japanese census 2010. We conclude that inter-prefecture migration for childcare is rare and it would not bias the estimate of the causal effect of childcare availability.

\subsection{Household Structure}

Our analysis so far takes the household structure as given, but women whose labor force attachment are strong might choose to form a three-generation household, rather than a nuclear household, because informal childcare may be available in three-generation households. Sasaki (2002) addresses this issue and estimates the effect of co-residence with the parents or in-laws on the wife's labor force participation. He instruments co-residence by whether the husband is the eldest son or not, because the traditional Japanese family value obligates the eldest son to reside with his parents. He finds that his OLS estimate for the effect of co-residence is essentially same as the IV estimate, implying that co-residence can be taken exogenous to the wife's labor force participation.

We have another concern that childcare availability may affect the household structure. For parents, a benefit of forming a three-generation household is that grand parents may provide informal childcare. If quality childcare is provided by the local government at an affordable price, the economic benefit of three-generation household decreases. Consequently, some parents may want to form a nuclear household for more privacy and independence.

If the growth of childcare availability increased the nuclear household share, childcare availability has a direct effect and an indirect effect through an increase in the nuclear household share. Note that the direct effect is positive while the indirect effect is negative. Our estimates for the causal effects capture the direct effect only, and we might over-estimate the overall effect of childcare availability.

To explore this issue, we regress 5-year changes in the nuclear household share on 5-year

\footnotetext{
${ }^{12}$ These six respondents also answer whether they moved from other prefectures or not and whether they knew or researched about childcare availability, but we are unable to report their answers to protect their privacy due to the number of observations being too few.
} 
changes in the capacity-population ratio. Table 5 reports the estimation results. Without any control, the coefficient is negative (see Model 1), but with year dummies, the coefficient is positive significant (see Model 2). Adding changes in age, husband's age and the employment rate, and the local unemployment rate has little effect on the coefficient (see Model 3). However, once we allow for a prefecture-specific trend by including prefecture dummies, the coefficient becomes insignificant. Note that the coefficient is rather precisely estimated with the standard error of 0.057 , which implies that the effects of childcare availability on the household structure is very small. Our data at hand and econometric model do not reject the hypothesis that childcare availability has no effect on the household structure.

Our own regression results and the finding by Sasaki (2002) suggest that our regression results are unlikely to be biased even though we take the household structure as exogenous.

Table 5: Childcare Availability and Nuclear Household Share

\begin{tabular}{lcccc}
\hline & Model 1 & Model 2 & Model 3 & Model 4 \\
\hline$\Delta$ Cap./U6-Pop. & $-0.143^{*}$ & $0.439^{* * *}$ & $0.392^{* * *}$ & 0.015 \\
& $(0.071)$ & $(0.083)$ & $(0.084)$ & $(0.057)$ \\
$\Delta$ Age & & & $0.052^{* *}$ & -0.005 \\
& & & $(0.019)$ & $(0.013)$ \\
$\Delta$ Husband's Age & & & $-0.064^{* * *}$ & 0.001 \\
& & & $(0.018)$ & $(0.012)$ \\
$\Delta$ Husband's \% Empl. & & & $0.277^{*}$ & $-0.192^{* *}$ \\
& & & $(0.107)$ & $(0.061)$ \\
$\Delta$ Unemp Rate & & & -0.327 & -0.227 \\
& & & $(0.255)$ & $(0.124)$ \\
(Intercept) & $0.040^{* * *}$ & $0.032^{* * *}$ & $0.033^{* * *}$ & $0.047^{* * *}$ \\
& $(0.002)$ & $(0.002)$ & $(0.005)$ & $(0.003)$ \\
Year FE & No & Yes & Yes & Yes \\
Prefecture FE & No & No & No & Yes \\
\hline $\mathrm{R}^{2}$ & 0.021 & 0.360 & 0.429 & 0.912 \\
Adj. R & 0.016 & 0.346 & 0.403 & 0.876 \\
Num. obs. & 188 & 188 & 188 & 188 \\
\hline$* * * p 0.001 *^{* *}<0.01 * *^{*}<0.05$ & & & &
\end{tabular}

Source: Census 1990-2005

Note: The dependent variable is 5-year changes in the nuclear household share among all households with children under 6 . Number of household is used as weight. 


\section{Growth of Maternal Employment Rate Across Regions}

Our analysis so far shows that childcare availability increases the employment rate of mothers of children under 6 in nuclear households and that the maternal employment rate is strongly affected by the household structure. In this section, we explore the implication of our findings for differences in the maternal employment growth across prefectures.

Table 6: Regional Differences

\begin{tabular}{|c|c|c|c|c|c|c|c|c|c|}
\hline & \multicolumn{3}{|c|}{ \% Employed } & \multicolumn{3}{|c|}{ Cap./U6-Pop. } & \multicolumn{3}{|c|}{ \% Nuclear HH } \\
\hline & 1990 & 2010 & Diff. & 1990 & 2010 & Diff. & 1990 & 2010 & Diff. \\
\hline All Prefs. & 0.341 & 0.412 & 0.070 & 0.248 & 0.338 & 0.089 & 0.712 & 0.865 & 0.153 \\
\hline Large Prefs. & 0.261 & 0.355 & 0.094 & 0.212 & 0.280 & 0.067 & 0.811 & 0.919 & 0.108 \\
\hline Small Prefs. & 0.409 & 0.470 & 0.061 & 0.279 & 0.397 & 0.118 & 0.628 & 0.810 & 0.181 \\
\hline
\end{tabular}

Source: Census 1990-2010

Note: Unit of observation is prefecture. Mean is calculated using the number of households with children under 6 in each year as weight. Large prefectures in terms of population density as of 2010 include Tokyo, Kanagawa, Saitama, Chiba, Aichi, Osaka, Hyogo, and Fukuoka.

Table 6 reports the maternal employment rate, the capacity-population ratio, and the nuclear household share across time and prefecture. The statistics are based on all two-parent households with children under 6 , regardless of whether they live with their parents or other relatives or not. Unit of observation is prefecture and the number of households are taken as weight. We separate prefectures in terms of population density as of 2010. The large prefectures include Tokyo, Osaka, Kanagawa, Saitama, Chiba, Aichi, Hyogo, and Fukuoka. The small prefectures include all other prefectures.

We compare the large and small prefectures in 1990. The maternal employment rate is only $26.1 \%$ in the large prefectures, while it is $40.9 \%$ in the small prefectures. This difference can be explained by childcare availability and the household structure at least partially. The ratio of childcare capacity to population of children under 6 is 0.212 in the large prefectures, while it is 0.279 in the small prefectures. Given our regression results above, the low childcare availability in the large prefectures can account for some of the difference in the maternal employment rate between the large and the small prefectures. The nuclear household share is $81.1 \%$ in the large prefectures, while it is significantly lower at $62.8 \%$ in the small prefectures. In 1990, the average maternal employment rate for nuclear households is $27.3 \%$, while it is as high as $51.1 \%$ for threegeneration household (not reported in the table). Given this large difference in the employment rate between nuclear and three-generation households, the difference in the nuclear household 
share accounts for some of the difference in the maternal employment rate between the large and small prefectures.

The changes from 1990 to 2010 are also substantially different across regions. The maternal employment rate grew at a faster pace in the large prefectures than the small prefectures (9.4 pt vs $6.1 \mathrm{pt})$. Accredited childcare became more available in both regions, but the capacity-population ratio grew at a slower pace in the large prefectures (0.067 vs 0.118$)$, which may be intuitive given that the supply shortage of childcare has been a major problem mostly in the metropolitan areas such as Tokyo. The nuclear household share increased in both regions, but the change is more strongly pronounced in the small prefectures than the large ones. Note that this change directly implies a significant drop in the share of three-generation households, because $99.8 \%$ of households are either nuclear or three-generation households.

We decompose the change in the maternal employment rate from 1990 to 2010 for each prefecture group using the method similar to the Oaxaca-Blinder decomposition. We estimate model parameters by applying the OLS with year and prefecture fixed effects (see Model 4 of Table 3) separately for each household type. The parameter estimates are used to decompose the overall change into the effect of the change in childcare availability, the change in the household structure, the change in the size of prefectures, and other factors. Depending on whether the base year for the decomposition is 1990 or 2010, four possible decompositions exist (see Appendix A.1 for details).

Table 7: Decomposition of Maternal Employment Growth from 1990 to 2010 (All Prefectures)

\begin{tabular}{lrrrr}
\hline \hline & Model 1 & Model 2 & Model 3 & Model 4 \\
\hline $\begin{array}{lrrr}\text { Overall Change } \\
\Delta \text { Empl. Rate }\end{array}$ & 0.070 & 0.070 & 0.070 & 0.070 \\
\hline $\begin{array}{l}\text { Decomposition } \\
\text { Childcare }\end{array}$ & 0.021 & 0.021 & 0.013 & 0.013 \\
Household Type & -0.028 & -0.029 & -0.011 & -0.011 \\
Prefecture Size & -0.008 & -0.007 & -0.007 & -0.007 \\
Other & 0.085 & 0.085 & 0.075 & 0.075 \\
\hline
\end{tabular}

Source: Census 1990-2010 and authors' calculation

Table 7 shows the decomposition for all prefectures. The growth of childcare availability modestly increased the maternal employment by 1.3-2.1 percentage points out of 7.0. The change in the household structure contributed negatively to the maternal employment growth. Namely, the increase in the nuclear household share decreased the maternal employment rate by 1.1-2.8 percentage points.

The driving factors of the maternal employment growth is considerably different between the 
Table 8: Decomposition of Maternal Employment Growth from 1990 to 2010 (Large Prefectures)

\begin{tabular}{lrrrr}
\hline \hline & Model 1 & Model 2 & Model 3 & Model 4 \\
\hline Overall Change & & & & \\
$\Delta$ Empl. Rate & 0.088 & 0.088 & 0.088 & 0.088 \\
\hline Decomposition & & & & \\
Childcare & 0.019 & 0.019 & 0.015 & 0.015 \\
Household Type & -0.017 & -0.017 & -0.007 & -0.007 \\
Prefecture Size & 0.000 & 0.000 & 0.000 & 0.000 \\
Other & 0.086 & 0.086 & 0.080 & 0.080 \\
\hline
\end{tabular}

Source: Census 1990-2010 and authors' calculation

prefecture groups. Table 8 shows the decomposition for the large prefectures. The growth of childcare availability in the large prefectures increased the maternal employment rate by 1.5-1.9 percentage points out of the overall change of 8.8 percentage points. The increase in nuclear households modestly decreased the maternal employment rate by $0.7-1.7$ percentage points. Because the nuclear household share was already high in 1990, the increase of nuclear households does not have a strong negative effect on the maternal employment rate.

Table 9: Decomposition of Maternal Employment Growth from 1990 to 2010 (Small Prefectures)

\begin{tabular}{lrrrr}
\hline \hline & Model 1 & Model 2 & Model 3 & Model 4 \\
\hline $\begin{array}{l}\text { Overall Change } \\
\Delta \text { Empl. Rate }\end{array}$ & 0.066 & 0.066 & 0.066 & 0.066 \\
\hline Decomposition & & & & \\
Childcare & 0.024 & 0.024 & 0.012 & 0.012 \\
Household Type & -0.039 & -0.040 & -0.015 & -0.015 \\
Prefecture Size & -0.003 & -0.002 & -0.003 & -0.002 \\
Other & 0.084 & 0.084 & 0.071 & 0.071 \\
\hline
\end{tabular}

Source: Census 1990-2010 and authors' calculation

Table 9 shows the decomposition for the small prefectures. The growth of childcare availability in the small prefectures increased the maternal employment rate by 1.2-2.4 percentage points out of the overall change of 6.6 percentage points. The increase in nuclear households significantly decreased the maternal employment rate by 1.5-4.0 percentage points in the small prefectures. Because three-generation household dissolved at a faster pace in the small prefectures, this effect is more strongly pronounced. 
Our decompositions shed light on the difference in the maternal employment growth across regions. As expected, childcare availability is an important determinant of the recent growth of maternal employment in both prefecture groups. However, dissolution of three-generation households lowered the maternal employment rate. This negative effect is particularly strong in the small prefectures, because three-generation households dissolved more quickly in the small prefectures than in the large prefectures.

Table 10: Decomposition of Growth of Capacity-to-Child-Population Ratio from 1990 to 2010

\begin{tabular}{ccccccccc}
\hline \hline & \multicolumn{2}{c}{ All Prefectures } & & \multicolumn{2}{c}{ Large Prefectures } & & \multicolumn{2}{c}{ Small Prefectures } \\
& Model 1 & Model 2 & & Model 1 & Model 2 & & Model 1 & Model 2 \\
\hline $\begin{array}{c}\text { Overall Change } \\
\Delta \text { Cap./U6-Pop. }\end{array}$ & 0.095 & 0.095 & & 0.068 & 0.068 & & 0.118 & 0.118 \\
\hline $\begin{array}{c}\text { Decomposition } \\
\text { Capacity }\end{array}$ & 0.027 & 0.023 & & 0.039 & 0.034 & & 0.017 & 0.014 \\
U6-Population & 0.068 & 0.071 & & 0.029 & 0.034 & & 0.101 & 0.105 \\
\hline
\end{tabular}

Source: Census 1990-2010 and authors' calculation

Finally, we decompose the growth of childcare availability. Our childcare availability index is given by the ratio of capacity to child population, and hence, a change in either capacity or child population affects the availability index. Our exercise here uncovers how the reasons for the growth of childcare availability differ between the two prefecture groups. In Appendix A.2, we describe two different ways to decompose the growth depending on the base year.

Table 10 shows the decomposition results. On average over all prefectures, the capacitypopulation ratio increased by 9.5 points. The increase in capacity accounts for $2.3-2.7$ points, while the decrease in population of children under 6 accounts for 6.8-7.1 points. While childcare became more available in both the small and the large prefectures, the reasons are very different between the two. In the large prefectures, about a half of the overall growth of childcare availability is explained by the increased capacity. In contrast, in the small prefectures, only about $15 \%$ of the growth of childcare availability is explained by the increased capacity. In both regions, the decrease in child population is an important factor for the better childcare availability, but it is even more important in the small prefectures.

\section{Conclusion}

We estimate the effects of childcare availability on maternal employment using prefecture panel data from the Japanese quinquennial census from 1990 to 2010. The first-difference estimator 
and OLS with year and prefecture fixed effects reveal that the employment rate of mothers in nuclear households increases with childcare availability, whereas that of mothers in three-generation household does not. The main result is robust to inclusion of a prefecture-specific trend and choice of econometric models. We also find that inter-prefecture migration for childcare is rare, which denies a possible simultaneity bias due to self-selection into prefectures where childcare is more available. Our analysis also suggests that controlling for prefecture fixed effects may be crucially important for identifying the causal effects of childcare availability at the prefecture level.

We take our estimates to examine how changes in childcare availability and the household structure affected the maternal employment growth. The growth of childcare availability increased the maternal employment rate by up to two percentage points in both large and small prefectures. Dissolution of three-generation households lowered the maternal employment rate in both prefecture groups. This negative effect is particularly strong and lowered the maternal employment rate by up to four percentage points in the small prefectures, because the share of three-generation households has been high. We also find that the growth of childcare capacity accounts for more than a half of the growth of childcare availability in the large prefectures. In contrast, in the small prefectures, the growth of childcare is mostly explained by the decreasing child population.

The main limitation of the current paper is that we do not examine detailed labor market outcomes such as wage, earnings, hours of work, and job type, due to lack of data. Understanding the effects of childcare availability on these outcomes will shed light on welfare consequences and opens the door to a cost-benefit analysis. We leave these interesting issues for future research.

\section{References}

ABE, Y. (2013): "Regional variations in labor force behavior of women in Japan," Japan and the World Economy, 28(0), 112 - 124.

AsAI, Y. (2012): "Parental Leave Reforms and the Employment of New Mothers: Quasiexperimental Evidence from Japan," University of Tokyo.

BERLINSKI, S., AND S. GALIANI (2007): “The effect of a large expansion of pre-primary school facilities on preschool attendance and maternal employment," Labour Economics, 14(3), 665 680.

Blau, D. M., And P. K. Robins (1988): “Child-Care Costs and Family Labor Supply," The Review of Economics and Statistics, 70(3), 374-81.

Brilli, Y., D. Del BocA, AND C. Pronzato (2013): "Does child care availability play a role in 
maternal employment and children's development? Evidence from Italy," Review of Economics of the Household, pp. 1-25.

Connelly, R. (1992): “The Effect of Child Care Costs on Married Women's Labor Force Participation," The Review of Economics and Statistics, 74(1), 83-90.

Gelbach, J. B. (2002): "Public Schooling for Young Children and Maternal Labor Supply," American Economic Review, 92(1), 307-322.

Higuchi, Y., T. MatsunRA, And K. SATO (2007): "Effects of Regional Factors on Fertility and Employment Continuity of Wives," RIETI Discussoin Paper No. 07-J-012 (in Japanese).

Kimmel, J. (1998): “Child Care Costs As A Barrier To Employment For Single And Married Mothers," The Review of Economics and Statistics, 80(2), 287-299.

LEE, G. H., AND S. P. LEE (2014): “Childcare availability, fertility and female labor force participation in Japan,' Journal of the Japanese and International Economies, 32(0), 71 - 85.

Lefebvre, P., And P. Merrigan (2008): "Child-Care Policy and the Labor Supply of Mothers with Young Children: A Natural Experiment from Canada," Journal of Labor Economics, 26(3), 519-548.

Michalopoulos, C., P. K. Robins, And I. GarfinKel (1992): “A Structural Model of Labor Supply and Child Care Demand," Journal of Human Resources, 27(1), 166-203.

Ministry of Health, L., AND Wealth (2009): "Summary of the Survey of Local Child Welfare Services," available from http://www.mhlw.go.jp/toukei/saikin/hw/jidou/09/dl/80.pdf.

SASAKI, M. (2002): "The Causal Effect of Family Structure on Labor Force Participation among Japanese Married Women," Journal of Human Resources, 37(2), 429-440.

Shigeno, Y., AND Y. OoKusa (1999): “The Effects of Childcare Policy on Fertility and Employment," Kikan Shakai Hosho Kenkyu, 35, 192-207 (in Japanese).

TABuchi, H. (2013): “Desperate Hunt for Day Care in Japan,” New York Times, Online; Published February 26, 2013.

UnAYAma, T. (2011): "Compatibility of Marriage, Fertility, and Work and Childcare Availability," Nihon Keizai Kenkyu, 65, 1-22 (in Japanese).

Yamaguchi, S. (2014): "Dynamic Effects of Parental Leave Policy on Female Employment," McMaster University. 


\section{A Decompositions}

\section{A.1 Decomposition of the Growth of Maternal Employment Rate}

We estimate a linear regression model with year and prefecture fixed effects by OLS separately for each household type. We assume that the parameters are time-invariant. The predicted maternal employment rate of prefecture $i$ for household type $j$ in year $t$ is given by

$$
\hat{Y}_{i j t}=X_{i j t} \hat{\beta}_{j}
$$

where $\hat{\beta}_{j}$ is the estimated parameter value for household type $j$. Let $I$ be the set of prefectures of interest (all, large, or small). Let $J$ be the set of all household types. The average employment rate in year $t$ is given by

$$
\bar{Y}_{t}=\sum_{i \in I} \sum_{j=J} \omega_{i j t} \hat{Y}_{i j t}
$$

where $\omega_{i j t}$ is the weight for household type $j$ in prefecture $i$ in year $t$.

Let $N_{i j t}$ is the number of type $j$ households in prefecture $i$ in year $t$. Define $N_{t}$ be the aggregate number of household in year $t, N_{t} \equiv \sum_{i \in I} \sum_{j \in J} N_{i j t}$. The weight $\omega_{i j t}$ for household type $j$ in prefecture $i$ in year $t$ is defined by

$$
\omega_{i j t} \equiv \frac{N_{i j t}}{N_{t}}
$$

Let $N_{i t}$ be the number of all households in prefecture $i$ in year $t$. The weight $\omega_{i j t}$ can be decomposed as the product of two weights,

$$
\begin{aligned}
\omega_{i j t} & =\frac{N_{i t}}{N_{t}} \cdot \frac{N_{i j t}}{N_{i t}} \\
& \equiv \omega_{i t} \cdot \omega_{i j t},
\end{aligned}
$$

where $\omega_{i t}$ is the weight for prefecture $i$ in year $t$ and $\omega_{i j t}$ is the weight for household type $j$ within prefecture $i$ in year $t$.

The average growth of the maternal employment rate is given by

$$
\begin{aligned}
\Delta \bar{Y}_{t} & =\sum_{i \in I} \sum_{j=J}\left(\omega_{i j t} \hat{Y}_{i j t}-\omega_{i j t-1} \hat{Y}_{i j t-1}\right) \\
& =\sum_{i \in I} \sum_{j=J}\left(\omega_{i j t} \hat{Y}_{i j t}-\omega_{i j t} \hat{Y}_{i j t-1}+\omega_{i j t} \hat{Y}_{i j t-1}-\omega_{i j t-1} \hat{Y}_{i j t-1}\right) \\
& =\sum_{i \in I} \sum_{j=J}\left(\Delta \hat{Y}_{i j t} \omega_{i j t}+\Delta \omega_{i j t} \hat{Y}_{i j t-1}\right),
\end{aligned}
$$


where the first term in the parenthesis is the effect of the growth of the maternal employment rate $\Delta \hat{Y}_{i j t}$ for a fixed weight $\omega_{i j t}$ and the second term is the effect of changes in weights $\Delta \omega_{i j t}$. We can equivalently decompose it as

$$
\Delta \bar{Y}_{t}=\sum_{i \in I} \sum_{j=J}\left(\Delta \hat{Y}_{i j t} \omega_{i j t-1}+\Delta \omega_{i j t} \hat{Y}_{i j t}\right)
$$

The change in the weight $\Delta \omega_{i j t}$ can be decomposed as

$$
\begin{aligned}
\Delta \omega_{i j t} & =\omega_{i t} \omega_{i j t}-\omega_{i t-1} \omega_{i j t-1} \\
& =\omega_{i t} \omega_{i j t}-\omega_{i t-1} \omega_{i j t}+\omega_{i t-1} \omega_{i j t}-\omega_{i t-1} \omega_{i j t-1} \\
& =\Delta \omega_{i t} \omega_{i j t}+\Delta \omega_{i j t} \omega_{i t-1},
\end{aligned}
$$

where the first term on the right hand side is the effect of changes in the prefecture size and the second term is the effect of changes in the household structure. We can equivalently decompose it as

$$
\Delta \omega_{i j t}=\Delta \omega_{i t} \omega_{i j t-1}+\omega_{i t} \Delta \omega_{i j t}
$$

The change in the maternal employment rate for household type $j$ in prefecture $i$ in year $t$ is given by

$$
\begin{aligned}
\Delta \hat{Y}_{i j t} & =\Delta X_{i j t} \beta_{j} \\
& \equiv \Delta C C A_{i t} \beta_{j}^{C C A}+\Delta X_{i j t}^{O} \beta_{j}^{O}
\end{aligned}
$$

where $\Delta C C A_{i t}$ is a change in the childcare availability index in prefecture $i$ in year $t$ and $\Delta X_{i j t}^{O}$ is changes in other covariates for household type $j$ in prefecture $i$ in year $t$.

We have the following four equivalent ways to decompose the average growth in the maternal employment rate,

$$
\begin{aligned}
\Delta \bar{Y}_{t} & =\sum_{i \in I} \sum_{j=J}\left(\Delta C C A_{i t} \beta_{j}^{C C A} \omega_{i j t}+\Delta X_{i j t}^{O} \beta_{j}^{O} \omega_{i j t}+\Delta \omega_{i t} \omega_{i j t} \hat{Y}_{i j t-1}+\Delta \omega_{i j t} \omega_{i t-1} \hat{Y}_{i j t-1}\right) \\
& =\sum_{i \in I} \sum_{j=J}\left(\Delta C C A_{i t} \beta_{j}^{C C A} \omega_{i j t}+\Delta X_{i j t}^{O} \beta_{j}^{O} \omega_{i j t}+\Delta \omega_{i t} \omega_{i j t-1} \hat{Y}_{i j t-1}+\Delta \omega_{i j t} \omega_{i t} \hat{Y}_{i j t-1}\right) \\
& =\sum_{i \in I} \sum_{j=J}\left(\Delta C C A_{i t} \beta_{j}^{C C A} \omega_{i j t-1}+\Delta X_{i j t}^{O} \beta_{j}^{O} \omega_{i j t-1}+\Delta \omega_{i t} \omega_{i j t} \hat{Y}_{i j t}+\Delta \omega_{i j t} \omega_{i t-1} \hat{Y}_{i j t}\right) \\
& =\sum_{i \in I} \sum_{j=J}\left(\Delta C C A_{i t} \beta_{j}^{C C A} \omega_{i j t-1}+\Delta X_{i j t}^{O} \beta_{j}^{O} \omega_{i j t-1}+\Delta \omega_{i t} \omega_{i j t-1} \hat{Y}_{i j t}+\Delta \omega_{i j t} \omega_{i t} \hat{Y}_{i j t}\right)
\end{aligned}
$$


For all of the four models, the first term in the parenthesis is the effect of changes in childcare availability $\triangle C C A_{i t}$, the second term is the effect of other covariates $\Delta X_{i j t}$, the third term is the effect of changes in prefecture size $\Delta \omega_{i t}$, and the fourth term is the effect of the household structure $\Delta \omega_{i j t}$.

\section{A.2 Decomposition of the Growth of Capacity-Population Ratio}

Let $C_{i t}$ be the childcare capacity in prefecture $i$ in year $t$. Let $N_{i t}^{C}$ be the population size of children under 6 in prefecture $i$ in year $t$. Our childcare availability index $C C A_{i t}$ is given by

$$
C C A_{i t}=C_{i t} / N_{i t}^{C}
$$

The growth of childcare availability index is

$$
\Delta C C A_{i t}=C C A_{i t}-C C A_{i t-1} .
$$

We can decompose the growth as follows,

$$
\begin{aligned}
\Delta C C A_{i t} & =\frac{C_{i t}}{N_{i t}^{C}}-\frac{C_{i t-1}}{N_{i t-1}^{C}} \\
& =\frac{C_{i t}}{N_{i t}^{C}}-\frac{C_{i t-1}}{N_{i t}^{C}}+\frac{C_{i t-1}}{N_{i t}^{C}}-\frac{C_{i t-1}}{N_{i t-1}^{C}} \\
& =\frac{\Delta C_{i t}}{N_{i t}^{C}}+\frac{C_{i t-1}}{\Delta N_{i t}^{C}},
\end{aligned}
$$

where the first term on the right hand side is the effect of capacity growth and the second term is the effect of the growth of child population. An alternative way to decompose the growth of the childcare availability index is

$$
\Delta C A_{i t}=\frac{\Delta C_{i t}}{N_{i t-1}^{C}}+\frac{C_{i t}}{\Delta N_{i t}^{C}} .
$$

